\title{
Control of Polymer Quality in Gas Phase Polyethylene Reactors Using Feed Flow Rates
}

\author{
Al-haj Ali M. and Ali Emad ${ }^{+}$
}

\begin{abstract}
Nonlinear model predictive control algorithm (NLMPC) is used for the on-line control of polymer molecular weight distribution. The control of chain-length distribution is achieved by selecting a collection of points in the distribution and using it as set points for the control algorithm. NLMLPC is implemented in online fashion to manipulate both the hydrogen and monomer feed rates. The simulation investigated the control algorithm performance for set point tracking conditions. The results demonstrate that the control algorithm can regulate the entire molecular weight distribution with high computational efficiency and minimum steady state error even in the presence of modeling errors.
\end{abstract}

Index Terms-Molecular weight distribution, nonlinear model predictive control, Kalman filter, polymerization reactor control, fluidized bed reactor, polyethylene

\section{INTRODUCTION}

Today, polymers are widely used for various products. Each usage requires different specifications for the polymers In order to satisfy these demands, companies produce many different grades of polymer. These grades have a different Molecular weight distribution (MWD), average molecular weight, or (and) a different polydispersity index. MWD is considered as one of the fundamental properties that determines polymer properties and thus its applications. Therefore, it is important to monitor and control MWD during the industrial production of polymers. A significant amount of research has been done in the area of control, monitoring and modelling of polymerization reactors; excellent reviews have been given by several researchers [14]. A careful study of previous works, with focus on the description of polymer MWD, results in the following conclusions:

Theoretically, any desired form of the MWD can be produced by appropriate fractionation of very broad molecular weight polymer or blending narrowly distributed polymers. Fractionation is not employed because of different reasons; the main reason is the extremely high cost of this operation [5]. Blending is not widely used, because most of the catalysts produce widely-distributed polymers. Moreover, producing polymers, which are mixed on a microscale level, requires a melting process. This is not economical.

All the alternative techniques rely on mixing of different polymer materials at the molecular level. Different methods were examined for this purpose. For example, a mixture of different metallocenes is used [6-11] or a hybrid catalyst of

\section{Al-haj Ali M., and Ali Emad +}

Chemical Engineering Department, King Saud University P.O.Box: 800, 11421 Riyadh, Saudi Arabia

+ corresponding author, email: amkamal@ksu.edu.sa
Ziegler-Natta and metallocene catalysts in a one stage process [12-14]. Another approach is use a cascade of reactors, where each reactor is operated at different polymerization conditions (usually hydrogen concentration). This technology is already commonly used with ZieglerNatta catalysts. It has the advantage that only one optimized catalyst is required for the production of various grades. The desired molecular weight distribution and co-monomer distribution are designed by the process. This method is subject to high operational costs [15]. Moreover, it is pointed out that the overall process has a low throughput since two serial processes are employed [16]. The third approach is to vary the polymerization conditions, specially the concentration of chain transfer agents, in a single reactor. This approach has the advantage of requiring a single reactor that simplifies process design and reduces the operational costs. Furthermore, the periodic operation of continuous chemical reactors can improve the performance of the reacting system and allow better design and control of the molecular weight distribution in a single reactor $[5,17]$. An example of a process with variation of process conditions is the multizone reactor developed by Basell [18, 19]. The implementation of a single reactor, compared to implementing two reactors, improves polymer homogeneity and assures that the ratio of both polymer products in each particle is equal to the overall ratio of these products. However, the dynamic operation of the polymerization reactor is difficult and it is subject to appreciable production of off-specification products and/or other operational problems.

In this paper the issue of broadening the MWD via the third approach will be further investigated. The idea to assess the effect of using both the monomer and hydrogen feed rates to alter the molar ratio (X). The scope of this work is to investigate the production of polyethylene, in a fluidized bed reactor, with a well-defined molecular weight distribution using nonlinear model predictive controller (NLMPC).

\section{PROCESS MODEL}

In fluidized-bed polyethylene reactors, the copolymerization of ethylene and -olefin monomers is carried out using a multi-site Ziegler-Natta catalyst, which consists of three different types of active sites. Each active site produces polymer with molecular weight distribution that can be described by Schulz-Flory distribution. The polyethylene reactor process is depicted in Fig 1. The process model was developed in [20]; modifications made in this model were described in [21]. The detailed description of the model and its parameters are not repeated here.

\subsection{Molecular Weight Distribution Model}


The instantaneous molecular weight distribution for each type of active sites can be described by Flory-Schulz exponential function [22]

$$
y_{j}^{d}=j \cdot q^{2} \cdot \exp (-j \cdot q)
$$

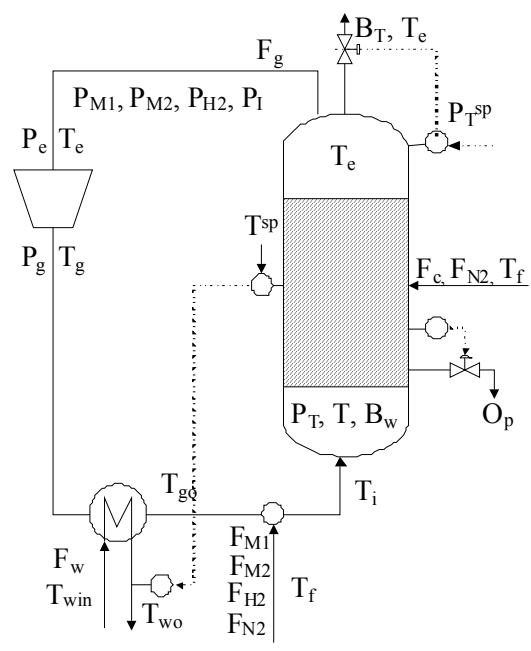

Fig. 1. Polyethylene reactor

with $q$ is the termination probability, $j$ is the number of repeating units and $y_{j}^{d}$ instantaneous weight distribution. As assumed above, the catalyst consists of three different active sites and the distribution of the polymers produced by each site type can be represented by Flory's most probable distribution. Thus, the overall distribution of the produced polymer can be calculated by the weighted sum of the three distributions as given below

$y_{j, \text { ins }}=\sum_{i=1}^{3} w_{i} \cdot\left(y_{j}^{d}\right)_{i}$

where $\mathrm{y}_{\mathrm{j}, \text { ins }}$ is the overall instantaneous molecular weight distribution, and $w_{i}$ is the mass fraction of each site. The molecular weight distribution of the polymer accumulated in the reactor after a certain polymerization time can be calculated using the following equation:

$$
\frac{d y_{j}}{d t}=\frac{O_{p} \cdot\left(y_{j, i n s}-y_{j}\right)}{B_{w}}
$$

here $y_{j}$ is the cumulative molecular weight distribution, $\mathrm{O}_{p}$ is polymer production rate and $\mathrm{B}_{\mathrm{w}}$ is mass of polymer in the reactor bed. Finally the GPC reading of the MWD is calculated by the following equation:

$$
G P C=j \cdot y_{j} \cdot \ln (10)
$$

\section{ON-LINE NLMPC ALGORITHM}

In this work, the structure of the MPC version developed by [23] that utilizes directly the nonlinear model for output prediction is used. A usual MPC formulation solves the following on-line optimization:

$$
\begin{gathered}
\min _{\Delta u\left(t_{k}\right), \ldots, \Delta u\left(t_{k+M-1}\right)} \sum_{i=1}^{\mathrm{P}}\left\|\Gamma\left(y\left(t_{k+i}\right)-R\left(t_{k+i}\right)\right)\right\|^{2}+ \\
\sum_{\mathrm{i}=1}^{\mathrm{M}}\left\|\Lambda \Delta u\left(t_{k+i-1}\right)\right\|^{2}
\end{gathered}
$$

subject to

$$
A^{T} \Delta U\left(t_{k}\right) \leq b
$$

For nonlinear MPC, the predicted output, $y$ over the prediction horizon $P$ is obtained by the numerical integration of:

$\frac{d x}{d t}=f(x, u, t)$

$y=g(x)$

from $t_{k}$ up to $t_{k+P}$ where $x$ and $y$ represent the states and the output of the model, respectively. The symbols $\|$. $\|$ denotes the Euclidean norm, $k$ is the sampling instant, $\Gamma$ and $\Lambda$ are diagonal weight matrices and $\mathrm{R}=[\mathrm{r}(\mathrm{k}+1) \ldots \mathrm{r}(\mathrm{k}+\mathrm{P})]^{\mathrm{T}}$ is a vector of the desired output trajectory. $\Delta \mathrm{U}\left(\mathrm{t}_{\mathrm{k}}\right)=\left[\begin{array}{lll}\Delta \mathrm{u}\left(\mathrm{t}_{\mathrm{k}}\right) & \ldots \Delta \mathrm{u}\left(\mathrm{t}_{\mathrm{k}+\mathrm{M}-1}\right)\end{array}\right]^{\mathrm{T}}$ is a vector of $\mathrm{M}$ future changes of the manipulated variable vector $u$ that are to be determined by the on-line optimization. The control horizon (M) and the prediction horizon (P) are used to adjust the speed of the response and hence to stabilize the feedback behavior. $\Gamma$ is usually used for trade-off between different controlled outputs. The input move suppression, $\Lambda$, on the other hand, is used to penalize different inputs and thus to stabilize the feedback response. The objective function (Eq. 5 ) is solved on-line to determine the optimum value of $\Delta \mathrm{U}\left(\mathrm{t}_{\mathrm{k}}\right)$. Only the current value of $\Delta \mathrm{u}$, which is the first element of $\Delta \mathrm{U}\left(\mathrm{t}_{\mathrm{k}}\right)$, is implemented on the plant. At the next sampling instant, the whole procedure is repeated.

To compensate for modeling error and eliminate steady state offset, a regular feedback is incorporated on the output predictions, $\mathrm{y}\left(\mathrm{t}_{\mathrm{k}+1}\right)$ through an additive disturbance term. Therefore, the output prediction is corrected by adding to it the disturbance estimates. The latter is set equal to the difference between plant and model outputs at present time $k$ as follows:

$$
d(k)=y_{p}(k)-y(k)
$$

The disturbance estimate, $d$, is assumed constant over the prediction horizon due to the lack of an explicit means of predicting the disturbance. However, for severe modeling errors, or open-loop unstable processes the regular feedback is not enough to improve the NLMCP response. Hence, state or parameter estimation is necessary to enhance the NLMPC performance in the face of model-plant mismatch. In this 
work, Kalman filtering (KF) will be incorporated to correct the model state and thus, to address the robustness issue. Utilization of the NLMPC with KF requires adjusting an additional parameter, $\sigma$. More details on the integration of KF with the NLMCP algorithm are given elsewhere [23]. In addition to state estimation by KF, the predicted output will be also corrected by the additive disturbance estimates of Eqn. 9.

The main objective of the NLMPC is to control the entire MWD. It is also necessary to maintain acceptable polymer production rate. Process stability is another important issue which is handled through regulating the total gas pressure and the bed temperature. These two controlled variables are adapted via separate PI control loops. The design and tuning parameters of these loops are given elsewhere [21].

\section{REsults AND Discussion}

It is worth mentioning that determining input trajectories that provide the desired distribution is difficult as the final polymer quality is sensitive to hydrogen concentration (X) value and the mass of the produced polymer. In this sense, maintaining the desired MWD during process operation is even more challenging. In the presence of model-plant mismatch and/or when unmeasured disturbances enter the plant, the situation becomes more complex. The control objective here is to produce broad polyethylene with welldefined MWD starting from narrow distribution and maintain it there. The results of this case are shown in Figs. 2 and 3. Four manipulated variables, which are the monomer, hydrogen, nitrogen and catalyst flow rates, are used. The weighting factors for these inputs are $\Lambda=\left[\begin{array}{llll}0 & 0 & 20 & 50\end{array}\right]$. Four controlled variables, which represent specific points in the target MWD, are considered as shown by the dots in Fig 3. The weighting factor for all outputs is given the same value of $\Gamma=\left[\begin{array}{llll}1 & 1 & 1 & 1\end{array}\right] \times 100$. The lower limit for the manipulated variables is set to zero and the upper limit is set to twice their nominal values. The MWD target function contains 103 points, however only four points were selected as controlled outputs to reduce the computation effort consumed by the NLMPC calculations. The input horizon (M) and output horizon (P) are taken equal to 1 and 4, respectively. A sampling time of $1 \mathrm{hr}$ is used. Usually the GPC measurements are available at low frequency. Advanced measurement sensors that can provide measurements in the order of minutes are available but at high cost.

Fig. 3 demonstrates the ability of NLMPC to maintain the new set point for the polymer distribution with minor distortion in the distribution function. More interesting is the response of the manipulated variables as shown in Fig. 2. The resulted response of the manipulated variables is in the form of periodic functions. Long prediction and moving horizon capability of NLMPC helped the controller to understand the dynamic nature of the process to an extent that it produced cyclic input sequences. Moreover, Fig. 2 shows how the bleed flow rate $\left(\mathrm{B}_{\mathrm{T}}\right)$ and the cooling water inlet temperature $\left(\mathrm{T}_{\mathrm{w}}\right)$ varies by separate PI controllers to maintain the total pressure at $20 \mathrm{~atm}$ and the reactor temperature at $82{ }^{\circ} \mathrm{C}$. Note that the manipulated variables used by NLMPC are plotted in discrete form because the
NLMPC works in discrete time fashion. It is obvious from Fig. 2 that the production rate, Op, reduces to an average value of $2.65 \mathrm{~kg} / \mathrm{s}$. In fact NLMPC reduces the monomer feed rate which in turn affects the reaction rate adversely. Moreover, the purge stream grows whenever the controller steer the monomer feed to high values. The growth in purge flow is enforced by the PI controller to regulate the reactor pressure back to normal condition. Therefore, the large variation in the monomer feed rate has a negative impact on both the production rate and the bleed flow.
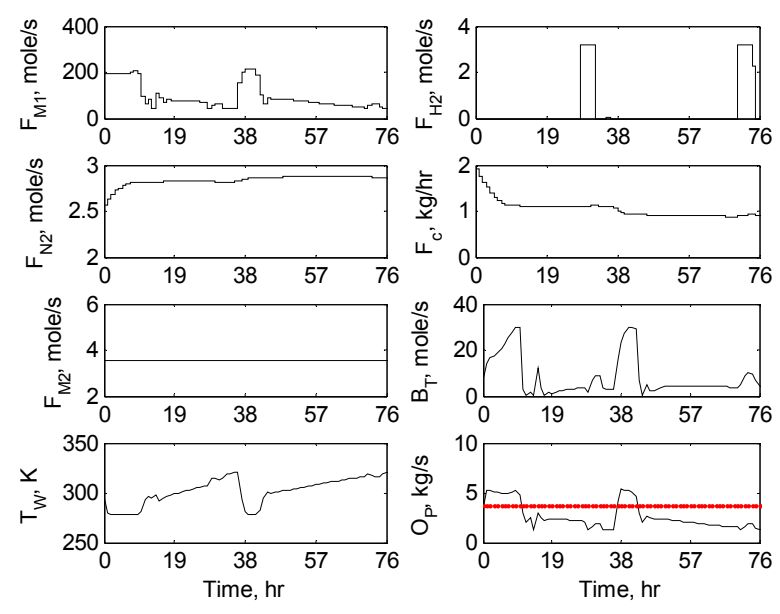

Fig. 2: Response of selected input and output variables using NLMPC for high average molecular weight case.

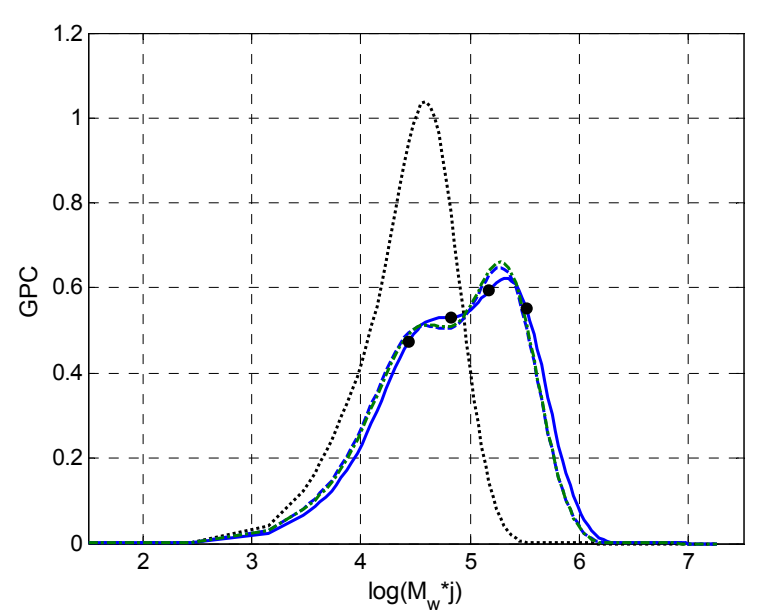

Fig. 3: MWD for high average molecular weight, Dotted line: initial distribution, solid: target, dashed: controlled distribution.

Next the control algorithm was tested for targeting another MWD which has a smaller average molecular weight. In this case, seven points on the GPC curve is taken as the controlled variables with their weights are fixed at $\Gamma=\left[\begin{array}{llllll}1 & 100 & 100 & 200 & 100 & 50\end{array}\right] \times 10^{2}$. The lower limit of $F_{M 1}$ is set to $40 \mathrm{~mole} / \mathrm{s}$ to keep high monomer concentration in the reactor. The value of the rest of the parameters remains the same as before. The simulation results are shown in Figs. 4 5. Evidently, NLMPC generated suitable periodic input sequences that produce MWD close to the desired one as shown in Fig. 5. The MWD suffered from minor distortion; however exact match of the target function is not necessary especially when we know that the relative error in GPC measurements is around $10 \%$. This outcome can be obtained 
at shorter simulation time. The small production rate is obvious from Fig. 4; in fact, the average production rate is found to be $2.42 \mathrm{~kg} / \mathrm{s}$. To improve the production rate, the latter is incorporated as a controlled variable in the NLMPC algorithm. Using $\gamma=0.1$ for the production rate, NLMPC managed to increase the polymer production to $2.86 \mathrm{~kg} / \mathrm{s}$ but with notable loss of the MWD. Results are not shown here for simplicity. Increasing the weight of the designated controlled output further will of course propagates the production rate but the MWD will depart away from the desired set point. Our investigation revealed the existence of trade-off between the production rate and broadening the MWD. Widening the distribution requires pronounced changes in hydrogen concentration inside the reactor. Increasing hydrogen concentration is achieved by feeding more hydrogen to the reactor this reduces ethylene polymerization rate and as a consequence reduces the overall production rate. Whereas, reducing hydrogen concentration is achieved by opening the vent [24] that allows hydrogen concentration in the reactor to escape, causing hydrogen concentration to fall quickly. Such reduction in the concentration affects positively on the production rate.
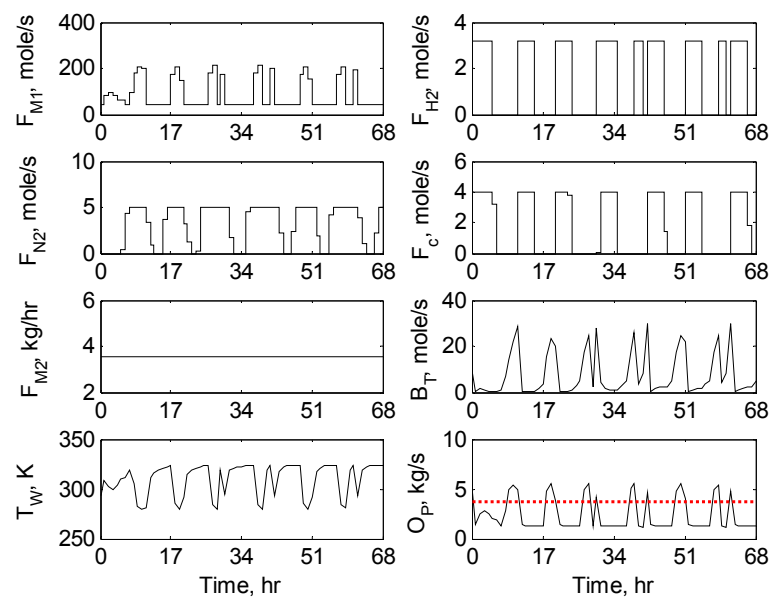

Fig. 4: Response of selected input and output variables using NLMPC for low average molecular weight case.

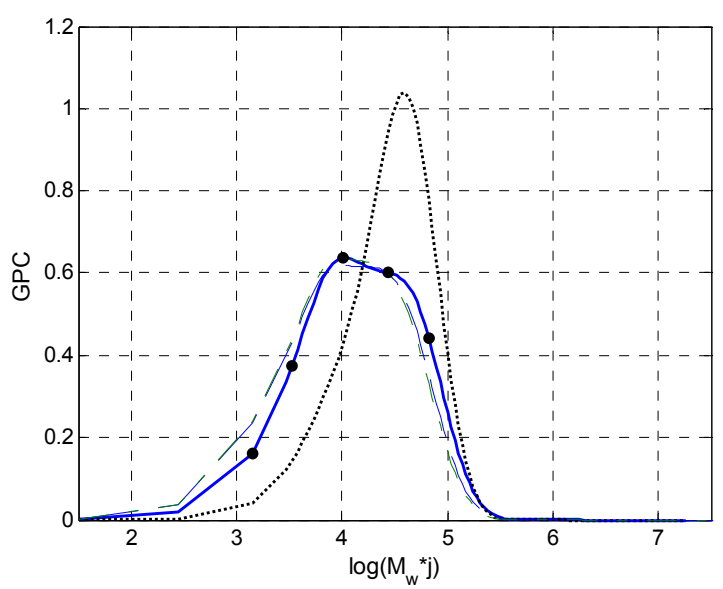

Fig. 5: MWD for low average molecular weight, Dotted line: initial distribution, solid: target, dashed: controlled distribution.
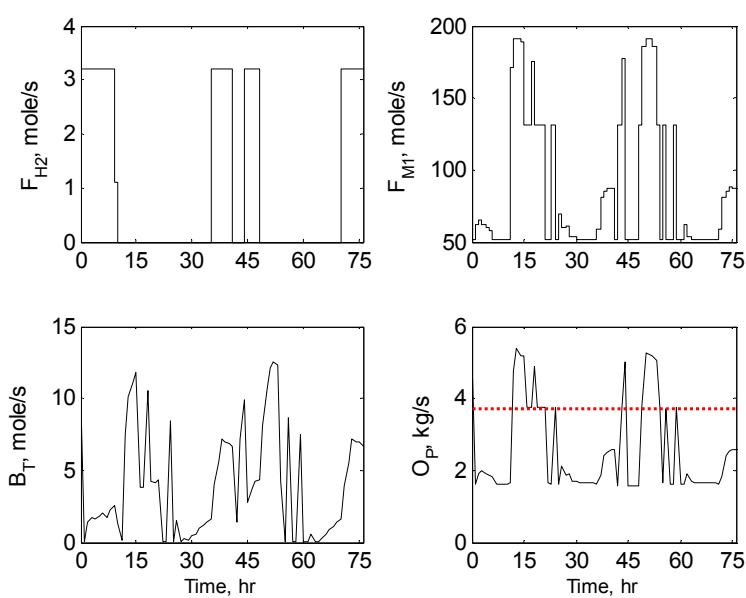

Fig. 6: Response of selected input and output variables using NLMPC for medium average molecular weight case.

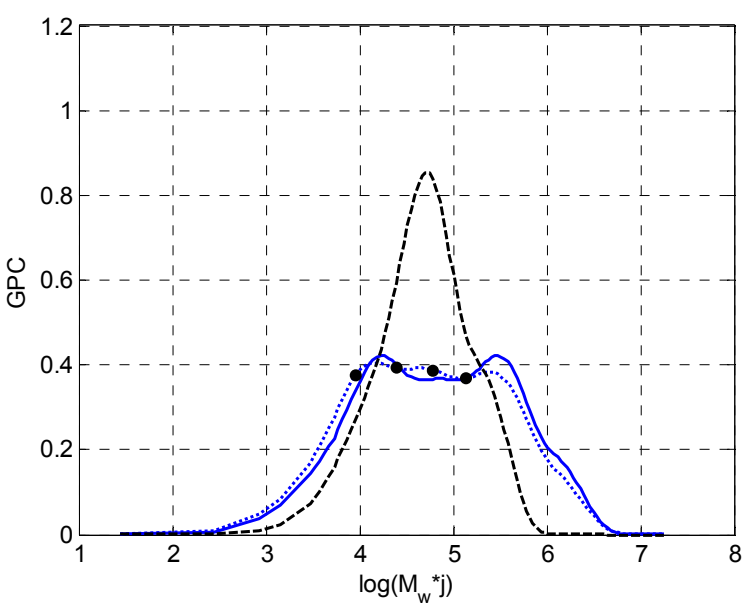

Fig. 7: MWD for medium average molecular weight, Dotted line: initial distribution, solid: target, dashed: controlled distribution.

The system is further tested for another target MWD that is much broader than the earlier two cases. Once again four representative points of the profile were chosen as set points. The results of the simulation are shown in Figure 6-7. The same simulation and NLMPC parameter values used previously is also employed in this case. Similar successful result in the sense of achieving the desired broad MWD is observed. However, the polymer production capacity is still influenced. Figure 6 illustrates how the production rate remains below the target value for long period of time reducing its average value to $2.55 \mathrm{~kg} / \mathrm{s}$.

The previous simulations are carried out assuming perfect model. However, this is not always true in real practice. To test the robustness of NLMPC in rejecting the effect of modeling errors, the simulation of targeting a MWD with higher average molecular weight is repeated with $-20 \%$ error in the reaction rate constant and $-20 \%$ error in the catalyst activity. It is expected that the parametric error will degrade the model predictions and consequently deteriorate the controller performance. The results are shown in Figs. 8 - 9. It is evident that NLMPC is able to keep good control performance despite minor loss of controller performance. The feedback characteristics of NLMPC and Kalman filter are the sources for the enhanced NLMPC performance in the last case where model-plant mismatch prevails. Similar 
robustness tests were carried out for the other MWD targets. Acceptable outcomes were observed in these cases. The simulation results were omitted here because no significant findings are reported.
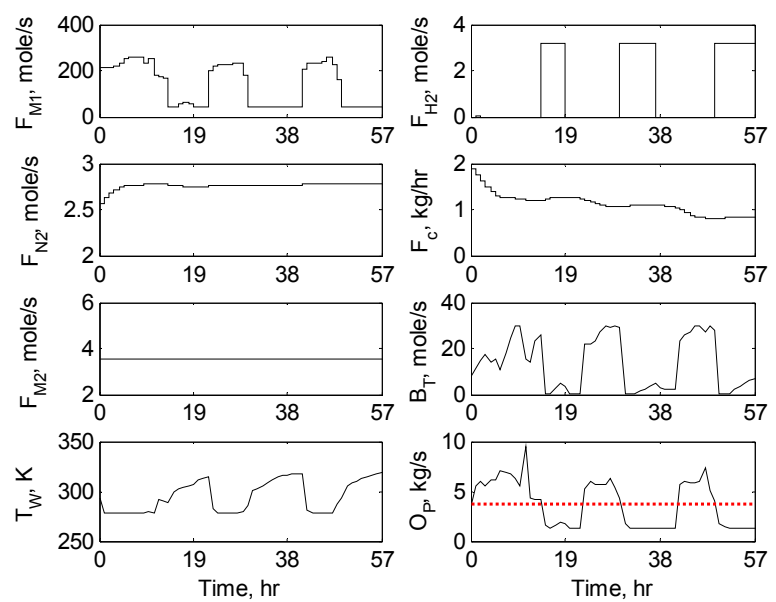

Fig. 8: Input and Output response for low average molecular weight in the presence of $-20 \%$ in catalyst activation and reaction rate constant.

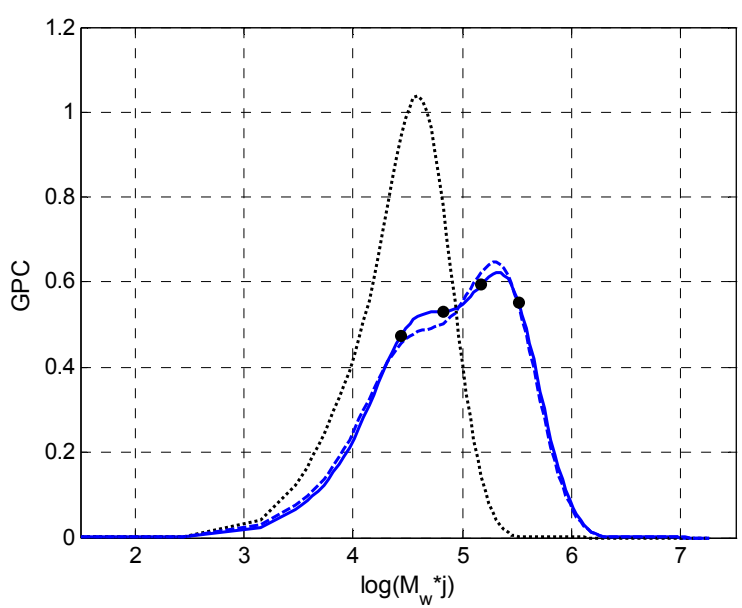

Fig. 9: MWD in the presence of $-20 \%$ error in catalyst activation and reaction rate constant. Dotted line: initial distribution, solid: target, dashed: controlled distribution.

The above three tests indicate that tracking a new polymer grade in terms of wide MWD is possible with the help of NLMPC. However allowing large variations in the monomer feed rate creates undesirable process operation. For example, the solid production is compromised when the monomer feed goes to minimum values. Furthermore, excessive lose of the gases can be generated when the monomer feed propagates considerably.

It is worth mentioning that controller performance could be improved more if the dynamics of hydrogen is faster. Since, hydrogen is not consumed in the reactor and large fluctuations in hydrogen concentration are required to broaden polymer distribution, improving controller performance would not be an easy task. This challenge can be solved using either a catalyst that is highly-sensitive to hydrogen as metallocenes or hydrogen consuming agent. The first approach depends on implementing a relatively new catalyst that is not widely used industrially [25]. The second approach still needs more investigation to prove its applicability for the studied process. Finally, note that venting is usually used to reduce hydrogen concentration, as described above, however; venting reactor contents is not an economical choice because monomer also escapes from the reactor. Nonetheless, no other choices are available.

\section{CONCLUSIONS}

In industrial applications, the molecular weight distribution of the produced polymer is usually measured using molecular weight averages and polydispersity index. In this article, we have presented an on-line MWD control technique to produce polymers with a target distribution in a fluidized-bed polymerization process. This strategy uses detailed polymerization process model, and Kalman filter to correct model states. A NLMPC controller is designed to control polymer MWD and polymerization process productivity. For the calculation of the MWD, selected points in polymer distribution curve are used as set-points for the controller that manipulates monomer, hydrogen, nitrogen and catalyst feed rates. To test the feasibility of the proposed MWD control technique, simulations have been carried out for ethylene gas-phase polymerization using conventional Zielger-Natta catalyst. The simulations suggest that the proposed control strategy can be useful new technique to control the MWD of polymer in continuous polymerization processes. The performance of the developed control algorithm can be improved more if the dynamic response of hydrogen concentration inside the polymerization reactor is less sluggish

\section{NOMENCLATURE}

Matrix of linear constraints

$b$

Linear constraints upper value

$B_{t} \quad$ Bleed flow rate, mole/s

$d \quad$ Disturbance estimates

$F_{c} \quad$ Catalyst flow rate. $\mathrm{kg} / \mathrm{s}$

$F_{M 1}, \quad F_{M 2}$, Monomer, co-monomer, hydrogen and

$F_{N}, F_{H} \quad$ nitrogen flow rate, mole/s

$j \quad$ The number of repeating units

$k \quad$ Sampling instant

$K F \quad$ Kalman filter

GPC Gel Permeation Chromatography

$M \quad$ Control horizon

MWD Molecular weight distribution

Op Polymer outlet rate, $\mathrm{kg} / \mathrm{s}$

$P \quad$ Prediction horizon

$q \quad$ chain termination probability

$T_{w} \quad$ Cooling water temperature, ${ }^{\circ} \mathrm{C}$

$R \quad$ Set point

$t \quad$ Time, s 
$u$

$w_{i}$

$X$

$y, y_{p}$

$y_{j}, y_{j}^{d}$

$\mathrm{y}_{\mathrm{j}, \text { ins }}$

$\Delta \mathrm{u}$

$\Delta \mathrm{U}$

$\Lambda$

$\Gamma$

$\sigma$

\section{REFERENCES}

[1] G. E. Elicabe, and G. R. Meira, "Estimation and Control in Polymerization Reactors." A Review. Polymer Engineering and Science, 8, 1988, 121-135.

[2] M., Embirucu, E. L. Lima, and J. C Pinto, "A Survey of Advanced Control of Polymerization Reactors." Polymer Engineering and Science, 36, 1996, 433-447.

[3] J. P. Congalidis, and J. R. Richards, "Process control of polymerization reactors: An industrial perspective." Polymer Reaction Engineering, 6, 1998, 71-111.

[4] J. R. Richards, and J. P. Congalidis, "Measurement and Control of Polymerization Reactors." Computers and Chemical Engineering, 30, 2006, 1447-1463.

[5] G. R. Meira, "Forced Oscillations in Continuous Polymerization Reactors and Molecular Weight Distribution Control. A Survey." Journal of Macromolecular Science-Reviews in Macromolecular Chemistry 20(2): 1981, 207-241.

[6] K. Heiland, and W. Kaminsky, "Comparison of Zirconocene and Hafnocene Catalysts for the Polymerization of Ethylene and 1Butene." Makromoleculare Chemie-Macromolecular Chemistry and Physics, 193, 1992, 601-610

[7] D. Beigzadeh, J. B. P. Soares and A. Hamielec. "Recipes for Synthesizing Polyolefins with Tailor-Made Molecular Weight, Polydispersity Index, Long-Chain Branching Frequencies, and Chemical Composition Using Combined Metallocene Catalyst Systems in a CSTR at Steady State." Journal of Applied Polymer Science, 71, 1998, 1753-1770.

[8] L. D'Agnillo, J. B. P. Soares and A. Penlidis. "A Critical Examination of Polyethylene Molecular Weight Distribution Control through the Combination of Soluble Metallocene/methylalumoxane Catalysts." Polymer International 47, 1998, 351-360.

[9] J.D. Kim, J.B.P. Soares, and G.L. Rempel, "Use of Hydrogen for Tailoring of the Molecular Weight Distribution of Polyethylene in a Bimetallic Supported Metallocene Catalyst System." Macromole. Rap. Comm. 19, 1998, 197-199.

[10] D. R. Loveday, and D. H. McConville, U.S. Patent 20010031695, 2001

[11] R. E. Murray, and S. Mawson, U.S. Patent 20010031697, 2001

[12] H. S. Cho, J. S. Chung and W. Y. Lee. "Control of Molecular Weight Distribution for Polyethylene Catalyzed over Ziegler-Natta /Metallocene Hybrid and Mixed Catalysts." J. of Molecular Catalysis A: Chemical 159, 2000, 203-213.

[13] T. E. Nowlin, S. D. Schregenberger, P. P. Shirodkarand G. O. Tsien. Patent US20020173601, 2002.

[14] E. S. Shamshoum, H. Chen, and L, Margarito, U.S. Patent 6653 254, 2003.
[15] J.B.P. Soares, and A. Penlidis, "Measurement, Mathematical Modeling and Control of Distribution of Molecular Weight, Chemical Composition and Long-Chain Branching of Polyolefins Made with Metallocene Catalysts", in Metallocene-Based Polyolefins: Preparation, Properties and Technology, J. Scheirs and W. kaminsky, Editors. 2000, Johan Wiley.

[16] G. Debras, and J. Dath U.S. Patent 6294 500, 2001

[17] R. S. Schiffino, U.S. Patent 6653 254, 1995

[18] M. Covezzi, and G. Mei. "The Multizone Circulating Reactor Technology", Chemical Engineering Science, 56, 2001, 4059-4067.

[19] J. L. Santos, J. M., Asua, de la Cal, J. C. "Modeling of Olefin GasPhase Polymerization in a Multizone Circulating Reactor", Ind. Eng. Chem. Res., 45, 2006, 3081-3094.

[20] K. B., McAuley, D. A. McDonald, and P. J. McLellan, "Effects of Operating Conditions on Stability of Gas-Phase Polyethylene Reactors." AIChE, 41, 1995, 868-879.

[21] Ali Emad, K. Al-Humaizi and A. Ajbar, "Multivariable Control of a Simulated Industrial Gas-Phase Polyethylene Reactor", Ind. Eng. Chem. Res., 42, 2003, 2349-2364,

[22] Y. V., Kissin, F. M. Mirabella, and C. C. Meverden, "Multi-Center Nature of Heterogeneous Ziegler-Natta Catalysts: TREF Confirmation.” Journal of Applied Polymer Science, 43, 2005, 43514362.

[23] E. M. Ali, and E., Zafiriou, Optimization-based Tuning of Non-linear Model Predictive Control with State Estimation. J. of Process Control, 3, 1993, 97-107.

[24] D. Lo, and W. H. Ray, "Dynamic Modeling of Polyethylene Grade Transition in Fluidized Bed Reactors Employing Nicel-Diimine Catalysts", Ind. Eng. Chem. Res., 45, 2006, 993-1008.

[25] P. Galli, and G. Vecellio, "Technology: Deriving Force Behind Innovation and Growth of Polyolefins." Progress in polymer Science, 26, 2001, 1287-1336. 